\title{
Lo Público y Lo Privado en la Modernización de La Gestión Sanitaria: Conceptos.
}

\author{
PEDRO MENDOZA \\ Profesor Auxiliar, Universidad Nacional Mayor de San Marcos Planificación y Administración en Salud
}

\begin{abstract}
RESUMEN:
El presente artículo presenta una revisión de conceptos en torno a lo público y lo privado en el proceso de modernización y reforma sanitaria actualmente en curso en el país. Para ello se hace una presentación de los conceptos de lo público y lo privado en los diversos planos funcionales de un sistema de salud, es decir en la función de usuario de los servicios, en la función de prestador de los mismos, y en la función del financiador. Se introduce asimismo al concepto de "combinación público-privado", empleando para ello ejemplos tanto de la experiencia internacional como de la experiencia nacional. Finalmente se proponen algunos criterios de evaluación de procesos de reforma en las funciones del sistema de salud a partir de la división de las funciones de compra y producción de servicios, y se examinan brevemente las condiciones para que la introducción de mecanismos de mercado y de mayor participación del sector privado en la prestación se dé mejorando la eficiencia y calidad de las acciones de salud, y preservando el principio de equidad del sistema de salud.
\end{abstract}

Palabras claves: Combinación público-privado, separación compra-producción, financiamiento, planificación, sistemas de salud.

\section{PUBLIC-PRIVATE IN THE MODERNIZATION OF THE HEALTH REFORM SUMMARY}

We present a review of concepts around public an private in the process of modernization and health sector reform currently ongoing in Perú. We begin with a general overview of the public and private concepts in differente functional levels of a generic health systems, namely, users function, provider function and financing function. We introduce then the concept of "public-private Mix" by using examples of international experience and also from national experiences. Finally, we propose some criteria for assessment of reform processes in health care systems when they include a purchaser-provider split, and we examine conditions to be taken in account if the introduction of market mechanisms and a higher private participation in health care provision is promoted, but preserving efficiency and quality of health care, and improving the equity principles of the system.

Key words: Public-private mix, purchaser-provider split, financing, planning, health care systems.

Esta presentación tiene por objetivo discutir los conceptos y la experiencia internacional en torno a lo público y lo privado, en gestión de servicios de salud y su re-examen a la luz de las tendencias modernas.

Para ello el artículo se compone de cuatro capítulos: El inicial, introductorio, propone un marco de análisis funcional del Sistema de Servicios de Salud, sobre el cual se estructuran los siguientes capítulos. En el segundo, se introduce los conceptos de Público y Privado en relación a las diversas funciones del Sistema de Salud y, en particular, el concepto de Combinación Público/Privado (*). En el tercer capítulo, se expone algunos lineamientos internacionales de modernización de la gestión sanitaria y.

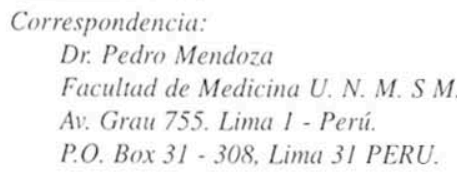

finalmente se proponen criterios para la evaluación de las formas de la Combinación Público-Privado, así como algunas condiciones y medios para que un relacionamiento diferente entre lo público y lo privado se de con la máxima preservación de la Equidad y Eficiencia de los Sistemas de Salud. 1. Análisis Funcional de los Sistemas de Salud.

Dentro de las numerosas perspectivas y modelos de análisis para examinar los Sistemas de Servicios de Salud, para este documento vamos a adoptar el Modelo de Reinhardt (1989), que propone una clasificación en cuatro grandes funciones para los agentes económicos en salud:

\footnotetext{
- La Función de Usuario

- La Función de Provisión

- La Función de Financiamiento

- La Función de Gobierno
}

El Término en inglés para este concepto es "Public-Private Mix", por lo cual algunos colegas lo traducen como "Mezcla Público-Privado", no obstante, coincidiendo con autores como Nabarro y otros, preferimos "Combinación" a "Mezcla". 
Como se observa en la fig. 1, cada uno de los agentes se vincula con los otros mediante relaciones de intercambio. La Función de Gobierno es una función inherente al Estado, en tanto direccionador y formulador de las políticas y marcos reguladores de funcionamiento del Sistema. En lo que sigue del artículo, vamos a examinar con mayor detenimiento las funciones de Usuario, Provisión, y Financiamiento.

\begin{tabular}{lll} 
& \multicolumn{1}{c}{ Pagos Directos } \\
USUARIOS & $<$ - & \\
& Atención de Salud &
\end{tabular}

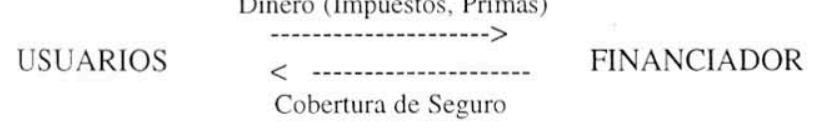

$\begin{array}{ccc} & \text { Pagos (Presupuesto, Tarifa) } \\ \text { FINANCIADOR } & & \\ & <-1 \text { Cuentas, Facturas } & \end{array}$

Gobierno = Establece el marco para el funcionamiento de los agentes económicos en salud

Fuente: Reinhardt, 1989

Fig 1.- Estructura del Sector Salud

Los Proveedores de Servicios son aquellas personas o instituciones que ofrecen los servicios al usuario. Un Consultorio Médico, un Policlínico o un Hospital son todos ejemplos de Proveedores. Los Proveedores pueden recibir un pago del usuario, ya sea por el total del servicio o por una fracción del mismo, asumiendo que el resto lo asumirá una institución a la que llamaremos "Financiador". Esto último es lo que sucede, por ejemplo, en un establecimiento del Ministerio de Salud en el cual se ha establecido el pago de tarifas para recuperación de costos.

El Financiador pagará al proveedor por sus servicios, ya sea a través de un Presupuesto, como es el caso de los Hospitales del Ministerio de Salud, o liquidando facturas, como es el caso de los Seguros Privados.

\section{Lo Público y Lo Privado}

Cada una de las funciones antes mencionadas puede ser llevada a cabo desde una perspectiva privada y desde una perspectiva pública.

\section{a) En la Función de Usuario.}

En la función de usuario, interesa diferenciar dos niveles de beneficio resultante de la atención: Un primer nivel, es el beneficio individual, resultante del usufructo de una atención por un individuo. En este caso, la utilidad social por el uso de tales servicios o atenciones es simplemente la sumatoria de las utilidades individuales. Así, el interés de la atención es esencialmente privado.

Un segundo nivel es el del beneficio colectivo, en el cual la utilidad social es mayor que la sumatoria de las utilidades individuales, es decir, el beneficio es colectivo, y por lo tanto, es este grupo de atenciones las que serían de interés público. Esto se da sobre todo con dos tipos de atenciones: Aquellas que involucran los denominados Bienes Públicos y las Atenciones Individuales con alta externalidad.
Los Bienes Públicos son aquellos bienes o servicios que poseen dos características: No Rivalidad (pueden ser usados por dos o más individuos simultáneamente) y No Exclusividad (No se puede evitar que una persona que desee utilizarlos lo haga). Este es el caso por ejemplo de la Educación Sanitaria por medios masivos, de las acciones de Control de Vectores, o de las acciones de salud ambiental y control de polución.

Las atenciones individuales con alta externalidad son aquellas que, si bien se ejercen y benefician directamente a una persona, por su naturaleza benefician en forma secundaria a otros individuos que no intervienen en dicha actividad. El control de la tuberculosis y de las enfermedades de transmisión sexual, así como las inmunizaciones, son ejemplos de tales atenciones, en las cuales el beneficio colectivo excede al de los directamente curados o vacunados.

A estas atenciones de beneficio colectivo es que algunos autores denominan "acciones de salud pública", si bien nosotros preferimos no usar tal denominación por las connotaciones más ámplias que el término tiene en algunas concepciones del estado.

b) En la Función de Financiamiento.

En este caso, lo público y lo privado se definen por la existencia o no de una identificación entre la calidad de aportante al sistema, y la capacidad para usufructuar el mismo. Cuando esta vinculación existe, decimos que el financiamiento es privado; cuando no existe, el financiamiento es público.

Los "Financiadores" habituales pueden ser un Ministerio o Secretaría de Salud, un Gobierno Regional o Municipal, un Instituto de Seguridad Social o una Empresa Privada de Seguros. De acuerdo al concepto antes presentado, en los dos primeros casos decimos que el financiamiento es público, pues no existe una vinculación individual entre el que paga (contribuyente) y el que usa el servicio, mientras que en los dos últimos casos el financiamiento es privado, en el sentido en que existe una correspondencia entre el haber pagado, las primas o cuotas del seguro, y el ser hábil para recibir el beneficio, es decir, gozar de cobertura, para lo cual se requiere un mecanismo de acreditación de derechos.

c) En la Función de Provisión.

En este caso, lo público y lo privado se definen en función de la propiedad del establecimiento u organización para efectos operacionales, definimos propietario como aquella instancia o nivel de la organización, que es capaz de decidir el cese de las operaciones de la misma.

d) El Concepto de Combinación Público-Privado

El Concepto de Combinación Público/Privado implica no sólo la consideración de dos sectores (Público y Privado) con grados variables de participación en determinados segmentos del mercado de servicios de salud, sino que es un intento de superar las limitaciones conceptuales de este análisis "sectorial", y reconocer no sólo la diversidad de los actores sociales involucrados en la atención de salud, sino también la variedad de relaciones que se puede desarrollar entre ellos. Así, este concepto puede permitir soluciones más creativas a los problemas crecientemente complejos que los sistemas de salud enfrentan.

En cuanto a la Provisión y Financiación, éstas son asumidas en forma convencional por un mismo agente; por ejemplo, el Ministerio de Salud es una Institución del Estado (Provisión Pública) que se financia a partir del Tesoro Público (Financiamiento Público) y que provee servicios directamente, siendo el prototipo de la prestación pública de servicios de salud, mientras un médico que instala su consultorio en forma independiente (Provisión 
Privada) y se financia a partir del cobro directo a los pacientes que atiende (Financiamiento Privado) sería un ejemplo de prestación privada de servicios de salud.

A Nivel Sistémico, se parte de la verificación que el patrón directo de Financiamiento-Provisión antes mencionado no tiene necesariamente que ser tal, sino que pueden haber formas mixtas en la cual el Financiamiento sea Público pero la Provisión Privada, como sucede por ejemplo cuando se contrata servicios a terceros, o que el Financiamiento puede ser Privado y la Provisión Pública, como cuando se instalan clínicas dentro de hospitales públicos.

En las fig. 2 y 3 se presenta estas modalidades en general, a nivel internacional y, con algunos ejemplos, a nivel nacional:

Provisión

\begin{tabular}{|c|c|c|c|}
\hline & & Pública & Privada \\
\hline \multirow{3}{*}{ 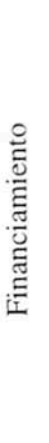 } & Público & $\begin{array}{l}\text { Servicios } \\
\text { Nacionales } \\
\text { de Salud }\end{array}$ & $\begin{array}{l}\text { Servicios } \\
\text { contratados a } \\
\text { proveedores privados }\end{array}$ \\
\hline & Privado & $\begin{array}{l}\text { Cobro } \\
\text { complementario } \\
\text { a los pacientes }\end{array}$ & $\begin{array}{l}\text { Proveedores privados } \\
\text { contratados por } \\
\text { seguros privados }\end{array}$ \\
\hline & & $\begin{array}{l}\text { Camas privadas } \\
\text { en Hospitales } \\
\text { Públicos }\end{array}$ & $\begin{array}{l}\text { Organizaciones de } \\
\text { Mantenimiento de } \\
\text { la Salud (HMOs) }\end{array}$ \\
\hline
\end{tabular}

(Fuente: Bennett, 1991)

Fig. 2.- Combinación Público-Privado a Nivel Internacional Provisión

\begin{tabular}{|c|c|c|c|}
\hline & & Pública & Privada \\
\hline \multirow{4}{*}{ 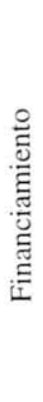 } & Público & & \\
\hline & & MINSA & $\begin{array}{l}\text { Servicios MINSA } \\
\text { contratados a } \\
\text { proveedores privados }\end{array}$ \\
\hline & Privado & $\begin{array}{l}\text { Cobro } \\
\text { complementario } \\
\text { a los pacientes }\end{array}$ & $\begin{array}{l}\text { Seguros Privados } \\
\text { que pagan a } \\
\text { Médicos privados }\end{array}$ \\
\hline & & IPSS - Directo & IPSS - PAAD \\
\hline
\end{tabular}

Fig. 3.- Combinación Público-Privado: Algunos ejemplos en el Perú

Podemos hablar, por lo tanto, de una combinación Público-Privado para la provisión de servicios, sin alterar los patrones de financiamiento, o podemos hablar de una combinación Público-Privado para el Financiamiento de Servicios, sin alterar los patrones de provisión de los mismos, o en una combinación simultánea en ambas funciones. En cualquier caso, el punto de partida es importante, pues en algunos países, como es el caso de China y los países del Este Europeo, hablar de Combinación Público-Privado es pasar de Servicios, tipo Públicos a una mayor participación del Sector Privado, mientras que en otros modelos, como en la propuesta Clinton para la Reforma de la Salud en Estados Unidos, se trata de incrementar la participación pública y una mayor regulación de la actividad privada.

\section{Tendencias Internacionales en la Gestión de los Servicios de Salud}

En los últimos cinco años se vive a nivel mundial una efervescencia de iniciativas, propuestas y experiencias, desde agencias internacionales, gobiernos y grupos académicos, que están llevando a la introducción de cambios significativos en la forma de entender la gestión de los servicios de salud.

Vamos a presentar los lineamientos de tres de ellas, que consideramos bastante ejemplificadoras de esta tendencia:

\section{a) La síntesis de Stewart y Walsh (').}

Stewart y Walsh, profesores de gestión pública en Birmingham, Reino Unido, publicaron en 1992 un artículo en el cual sintetizan los cambios que se venían dando a nivel mundial en la gestión de los servicios públicos como:

* Separación entre los roles de provisión y financiamiento.

* Crecimiento de arreglos contractuales.

* Mayor atención en el rendimiento y la productividad.

* Flexibilidad en las remuneraciones y las retribuciones a instituciones.

* Creación de cuasi-mercados.

* Visión del público como cliente.

* Incremento del rol regulador del Estado.

* Cambio en las culturas organizacionales.

b) La Propuesta del Banco Mundial $\left({ }^{2}\right)$ :

El Informe sobre el Desarrollo Mundial, en 1993, expresa la política sugerida por el Banco Mundial para una nueva relación entre los sectores público y privado y una reforma en la gestión en salud:

Esta propuesta se basa en tres lineamientos de política:

* Fomento de un entorno saludable:

- Políticas de crecimiento económico.

- Aumento de inversiones en educación, en especial para las niñas.

- Promoción de los derechos y situación de la mujer.

* Mejorar los gastos del gobierno en salud:

- Reducir los gastos en atención de nivel terciario.

- Financiar medidas de salud pública.

- Financiar un paquete de servicios clínicos esenciales

- Mejorar la eficiencia del gasto público y dar mayor participación al sector privado en la provisión de servicios.

* Promover la diversidad y la competencia:

- Promover un seguro social o privado para los servicios no comprendidos en el paquete básico.

- Impulsar a proveedores públicos y privados a competir en la prestación, con fondos públicos y privados.

- Obtener y difundir información sobre el costo y la efectividad de las intervenciones, así como sobre la calidad de los proveedores.

c) La Agenda Regional de OPS ( $\left.{ }^{3}\right)$

En 1995, la Organización Panamericana de la Salud publica un documento fruto de un trabajo interagencial que involucra al BID, Banco Mundial, CEPAL, OEA, OPS, UNFPA, UNICEF y USAID. En él menciona, entre los lineamientos operativos para el cambio en la gestión de los servicios, los siguientes: 
* Definición de paquetes básicos de atención de cobertura universal.

* Focalización de la acción del Estado en los grupos más vulnerables, con intervenciones costo-efectivas de alta externalidad sobre problemas prioritarios.

* Optimización de la combinación Público-Privado en la provisión de los servicios.

* Búsqueda de alternativas de financiamiento sectorial progresivas y compatibles con el balance macroeconómico.

* Redefinición del rol del Estado nacional, enfatizando las funciones de Gobierno y de apoyo a los gobiernos locales.

* Descentralización en la ejecución de programas.

* Incentivos al aumento de la equidad, eficiencia y calidad en la remuneración a los prestadores de los servicios.

* Participación de la sociedad en la formulación de políticas, financiación, gestión y evaluación.

4. Evaluación y Condiciones para una nueva relación público-privada. Se ha propuesto cuatro criterios para evaluar la pertinencia y éxito de las iniciativas de relación público-privada: Responsabilidad (Accountability), Eficiencia, Equidad, e Implementación de Prioridades en Salud (Mills, 1993).

a) Responsabilidad: Entendida ésta como la identificación de un ente jerárquicamente superior al cual se rinde cuentas (Accountability).

b) Eficiencia: Se entiende como la producción de servicios empleando las combinaciones de factores productivos que permitan los menores costos para la mejor calidad posible (Pierce, 1986).

c) Equidad: La entendemos como una proporcionalidad en la utilización de servicios de salud para los diversos grupos poblacionales en función de su necesidad.

d) Implementación de prioridades sanitarias: Determinadas por la autoridad de salud en el ámbito (Ministerio de Salud) en base al perfil epidemiológico y demográfico de la zona. Se comparará el perfil de servicios ofrecidos por el establecimiento con el propuesto o normado por la autoridad de salud.

Si bien la tendencia está ya establecida para un relacionamiento diferente entre la actividad pública y privada en salud, una planificación cuidadosa de este cambio es importante, tomando en cuenta la experiencia internacional existente y las peculiaridades de nuestro país.

La regulación del mercado en salud implica al menos tres elementos (Roberts, 1993):

* Promoción de un Mercado Competitivo. En nuestro país, esto es particularmente importante, dada la alta diversidad de realidades y la existencia de mercados de diverso grado de maduración. Esto implica que hay provincias del país con mercados altamente desarro- llados y que responderán de una manera rápida a los cambios introducidos; y otras provincias donde los cambios serán más lentos o no se darán. En todo caso, la promoción de la competitividad adoptando estrategias diferenciadas para cada realidad es un elemento esencial.

* Regulación y supervisión de contratos apropiados, efectivos y eficientes. La tecnología de contratación a terceros es aún débil desde el sector público. Si se trata de descentralizar, de promover eficiencia y de incentivar la participación de prestadores privados con financiamientos públicos o privados, el disponer de formas efectivas y eficientes de contratación en cada uno de los niveles efectores es otro elemento a desarrollar.

* Motivación y Monitoreo de los Agentes Económicos en Salud. La maduración de los mercados va a requerir un proceso de cuidadoso monitoreo y de medidas de promoción de la actividad privada, para que ésta pueda convertirse en un elemento dinámico y cuya participación efectivamente coopere para alcanzar los fines de equidad, calidad, eficiencia y efectividad del sistema.

Esta regulación del mercado, por lo tanto, implica responsabilidades del Gobierno tanto a nivel sistémico como a nivel corporativo:

a) Nivel Sistémico.

(1) Planificación y regulación de los Servicios

(2) Asegurar el financiamiento de los Servicios de Salud para los más vulnerables.

(3) Garantizar el acceso equitativo a los Servicios

(4) Financiar bienes públicos

(5) Control de externalidades negativas

b) Nivel Corporativo.

(1) Modalidad de Contrato/Remuneraciones

(2) Control de Medicamentos y Tecnología

(3) Información sobre rendimiento

(4) Desarrollo de estándares y vigilancia de la Calidad.

Si bien el desarrollo mayor de cada uno de estos puntos, su fundamentación, y el análisis técnico de las fortalezas y debilidades de cada una de las posiciones ameritaría ser puntos de agenda en sí mismos, hemos tratado de presentar en forma sintética algunos puntos de debate que consideramos importantes en un debate sobre la modernización de la gestión sanitaria.

\section{BIBLIOGRAFIA}

1) Stewart J, Walsh K. Change in the Management of Public Services. Public Administration 1992; 70: 499-518.

2) Banco Mundial. Informe sobre el Desarrollo Mundial 1993: Invertir en Salud, Washington, 1994.

3) OPS, Acceso Equitativo a los Servicios Básicos de Salud. Washington, 1995. 